\title{
A COMPARATIVE STUDY OF ACUTE POISONING IN NEPAL AT TERTIARY AND SECONDARY LEVEL HOSPITALS
}

\author{
Ghimire R H, Sharma S P*, Pandey K R
}

\section{ABSTRACT}

A study was done at Tribhuvan University Teaching Hospital (TUTH) and Bharatpur District Hospital (BDH) to find out the differences among the acute poisoning cases in those hospitals during 2000-2002. The study aimed to compare the acute poisoning cases in terms of some variables. It was a comparative study of all the acute poisoning cases admitted in the internal medicine department of TUTH and BDH during 2000-2002.

The numbers of acute poisoning cases were found to be more at BDH than at TUTH during the same period. Though the majority of the cases were female in both the hospitals, percentages of male poisoning cases were found to be more at BDH. The 16-25 age group was the predominantly affected group at TUTH, while it was below 15 age group at BDH. Pesticide poisoning cases were the commonest poisoning cases at TUTH whereas the numbers of rodenticides poisoning cases were predominant at $\mathrm{BDH}$. The use of drugs is significantly higher at TUTH. Deaths due to poisoning in TUTH were more than at BDH during the same period. The maximum number of poisoning cases occurred during the months of Bhadra- Mangsir. Owing to the higher rate of use of drugs as poison, the sale of drugs without prescriptions should be discouraged. Similarly the public should be well sensitized about the childhood poisoning.

\section{Key Words: Drugs, recovery, pesticides, rodenticides.}

\section{INTRODUCTION}

Suicide by acute poisoning is one of the leading causes of death. Acute poisoning is a common and urgent medical problem in all developed and developing countries ${ }^{1}$. Since 1960, suicide rate due to poisoning in young adults has increased in both sexes and the same trend is appearing among the elderly. Suicidal deaths due to poisoning in the fiscal year 1999/2000 were reported to be $31 \%$ of all the suicidal deaths. ${ }^{2}$ Patterns of poisoning in a society depend upon degree of intent of selfharm, socio-economic status and availability of poisonous substance. These factors are bound to change with the passage of time so does the poisoning pattern. However, there have been no studies in the past to compare the poisoning cases at different levels of health institutions at different geographical areas. With this background, this study is an attempt in revealing the different patterns of poisoning at a tertiary level hospital like TUTH and a district level hospital like Bharatpur district hospital during the same period of time.

\section{MATERIALS AND METHODS}

It was a comparative study of all the poisoning cases admitted at internal medicine department of TUTH and BDH (excluding those who attended and were discharged from emergency in both the centers) during 2000-2002. With the consent from the respective hospitals, the files of all the poisoning cases admitted in the internal medicine department of TUTH and BDH during 2000-2002 were reviewed. The information retrieved from the files was age, sex, month of poisoning, types

* Tribhuvan University Teaching Hospital, Maharajgunj, Kathmandu, Nepal.

Address for correspondence : Dr. Ram Hari Ghimire

Institute of Medicine, Maharajgunj, Kathmandu, Nepal.

Email: dr_rhg@yahoo.com 
Table I : Total number of poisoning cases and their Sex distribution

\begin{tabular}{c|c|c|c}
\hline & Male & Female & Total \\
\hline BDH & $47.4 \%(146)$ & $52.6 \%(162)$ & 308 \\
\hline TUTH & $42.66 \%(64)$ & $57.34 \%(86)$ & 150 \\
\hline
\end{tabular}

of poisons taken and the outcome of the treatment. Then the findings of these study variables at TUTH and BDH were compared with each other to show the differences among the poisoning cases at two health institutions.

\section{RESULT}

\section{The results of the study are as follows:}

Total number of poisoning cases and their Sex distribution:

Total number of poisoning cases admitted in the internal medicine department of TUTH (excludes those who attended and were discharged from emergency) during 2000-2002 was 150 while it was 308 at BDH (excluding those who attended and were discharged from emergency). There was higher percentage of male poisoning cases at BDH as compared to TUTH.

\section{Age distribution of the poisoning cases}

There was significant difference in the age composition of the poisoning cases at TUTH and BDH during the same period of 2000-2002. At TUTH most of the poisoning cases were of the age group 16-25 but at BDH maximum number of cases were below 15 years of age. The percentages of the cases in other age groups were almost similar.

\section{Month-wise distribution of the poisoning cases}

In both the centers, most of the cases were recorded during August to November followed by April to July.

\section{Types of the poison used by the poisoning cases}

$46.67 \%$ of the poisoning cases recorded at TUTH used Pesticides, especially organophosphates as poisoning material where as rodenticides were the most popular poison among the cases recorded at $\mathrm{BDH}$. Drugs were second only to pesticides at TUTH but at BDH pesticides were in the second place, drugs accounting only for $2.92 \%$ of the cases.

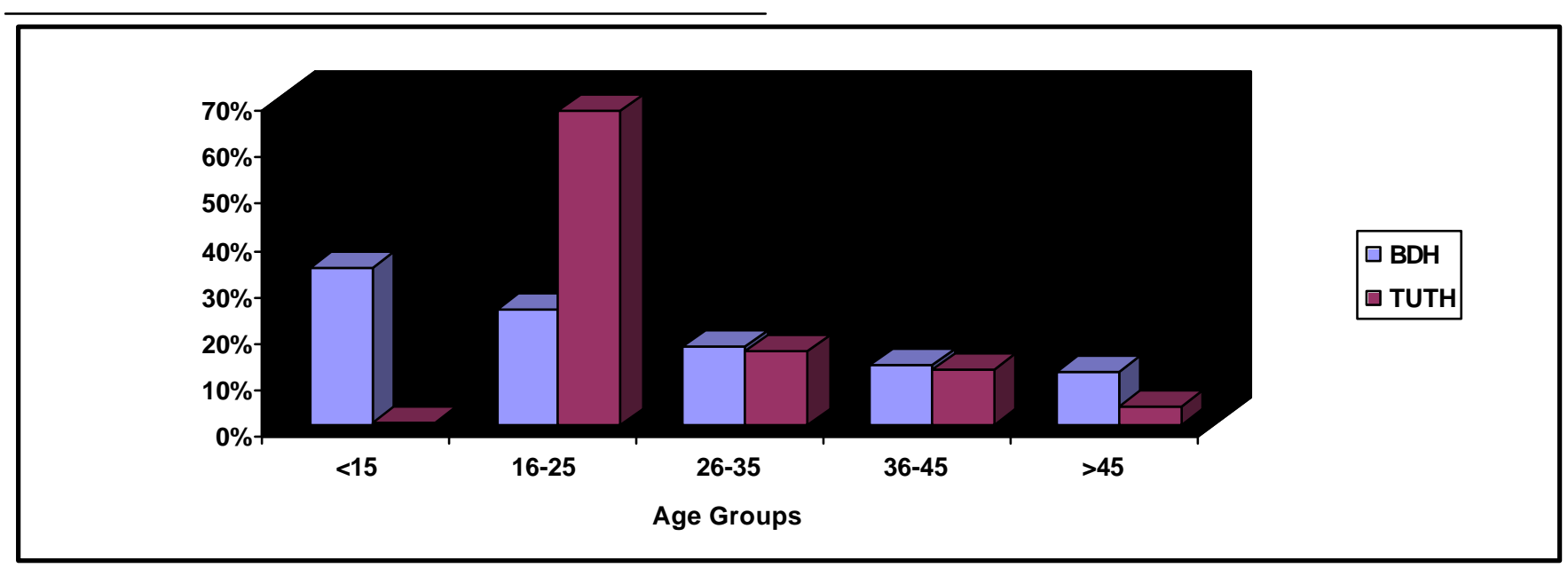

Fig. 1 : Age distribution of the poisoning cases

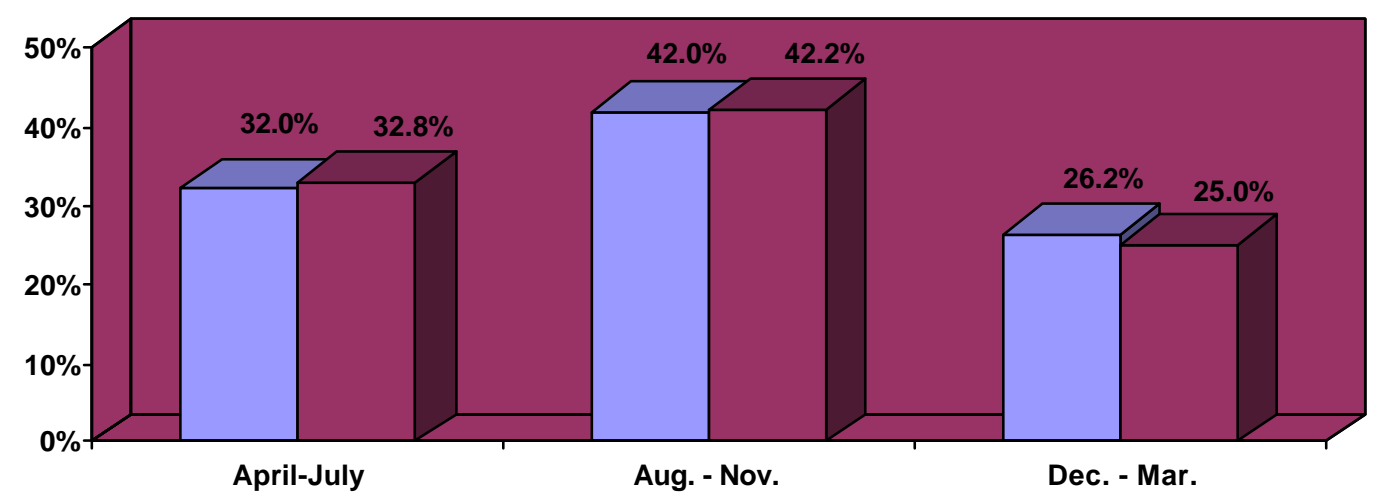




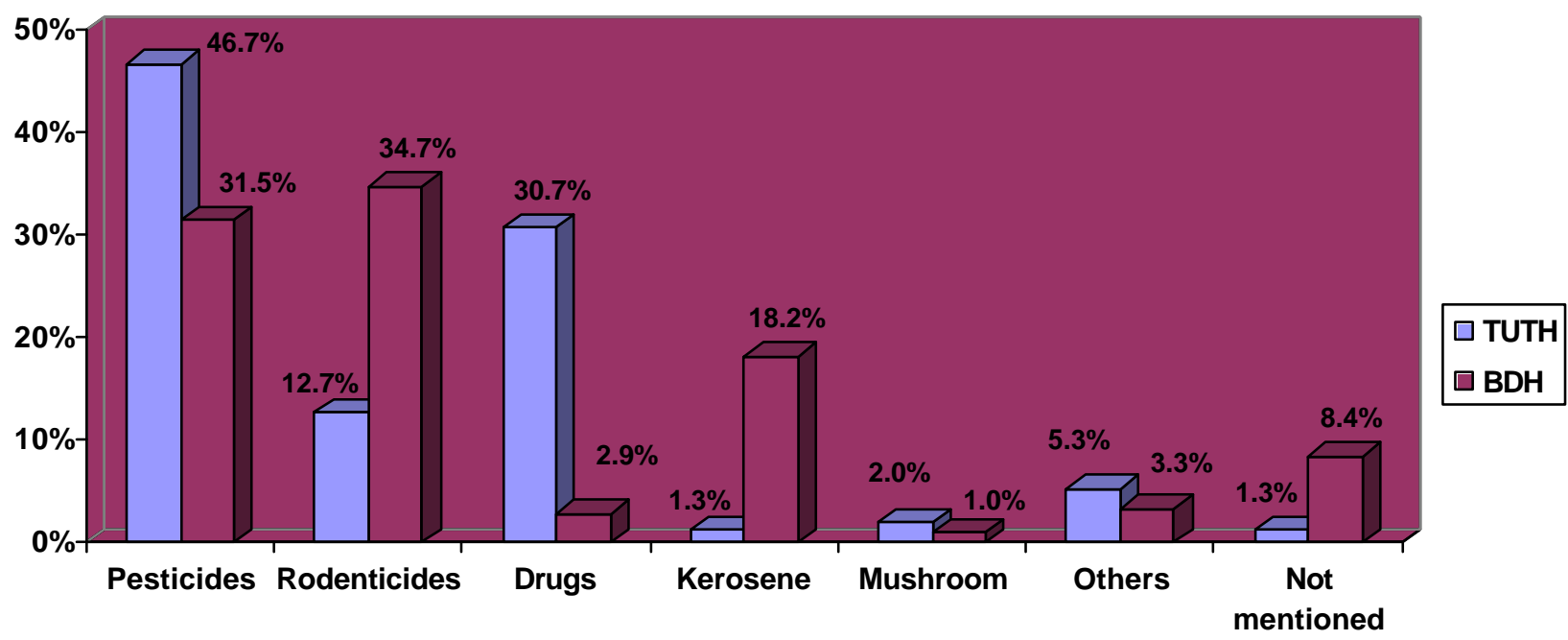

Types of poison

Fig. 3 : Types of the poison used by the poisoning cases

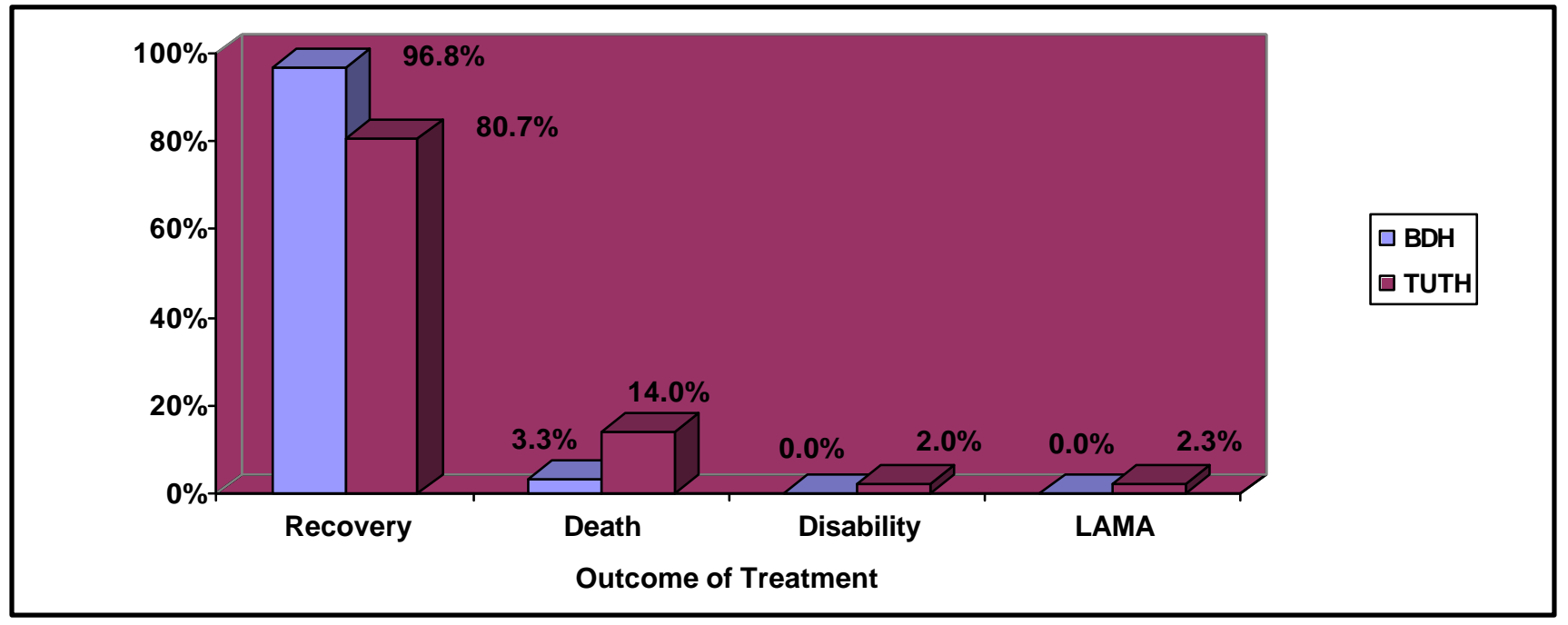

Fig. 4 : Outcome of Treatment of the poisoning cases

\section{*LAMA=Leave Against Medical Advice}

Other substances used as poison includes phenol, acid, alkali and organic herbicides.

\section{Outcome of Treatment of the poisoning cases}

The percentage of poisoning cases that ultimately succumbed to death was $14 \%$ at TUTH but it was comparatively very low at $\mathrm{BDH}$ as $96.75 \%$ of the cases got recovered.

\section{DISCUSSION}

Acute poisoning is the significant and common medical problem. The number of poisoning cases at TUTH is increasing every year ${ }^{4}$. Acute poisoning cases accounted for $1.1 \%$ of total cases presented at casualty department of TUTH during October 1995 to October $1996 .^{7}$

As there have been no studies on poisoning cases at $\mathrm{BDH}$ previously, the trend of poisoning cases whether increasing or decreasing could not be assessed. Our study has shown that the number of acute poisoning cases at BDH (308) outnumbered that of the TUTH (150) by more than two times during the same period. Unlike in the capital city where people have access to many tertiary care hospitals, people in a Terai district like Chitwan do not have many options. This might have lead to the concentration of the cases at BDH. In both health centers female constituted the predominant number of cases with female to male ratio of 1.10: 1 at $\mathrm{BDH}$ and 1.34: 1 at TUTH (excluding those who attended and were discharged from emergency in both the centers). This finding is consistent with several other studies conducted in the past in various hospitals of Nepal., ${ }^{3,4,5}$ At TUTH majority of the cases were in the age group 16-25 (67.34\%). This predominance is consistent with the age group reported from various studies in the past.? But at BDH maximum numbers of the cases were below 15 
years of the age. Lower number of below 15 cases at TUTH may be due to nearby Kanti Children Hospital $(\mathrm{KCH})$ dealing with the pediatrics poisoning cases. However, there have been no studies about the pediatric acute poisoning cases in $\mathrm{KCH}$ to assess the exact gravity of the problem. The higher number of pediatrics poisoning cases at BDH can be attributed to:

\section{Higher use of poisoning substances in Terai.}

Higher accidental exposure to poisons.

Inappropriate storage and disposal of poisonous materials. Inadequate attention of parents to their children.

Consistent with the studies done in the past at various hospitals of Nepal ${ }^{3,4,5,6,9}$ our study also found pesticides as the most commonly used poison at TUTH, but at BDH pesticides were second to rodenticides. Chitwan being primarily an agricultural district there is wide spread use of rodenticides to kill mice and squirrel so there is more and easy access to the rodenticides. The significantly higher number of the kerosene poisoning cases at BDH may be due to accidental ingestion of kerosene by below 15 children. The use of drug as poison is far more common at TUTH $(30.66 \%)$ than at BDH $(2.92 \%)$. The greater number of cases using drugs at TUTH may be related to higher rate of over the counter sale of drugs within the Kathmandu valley and better knowledge of people about the toxicities of the drugs.

Consistent with the finding of a study done by B.M Shrestha, ${ }^{10}$ in both hospitals the predominant number of cases were found during the months of August to November. The study shows that the majority of the cases recovered in both the centers. The higher percentage of deaths was recorded at TUTH. The higher percentage of accidental poisoning and higher percentage of people taking non-lethal poisons like kerosene at $\mathrm{BDH}$ account for this difference. This by no means implies the lower quality of health care at TUTH.

\section{CONCLUSION AND RECOMMENDATIONS}

? The number of poisoning cases was higher at BDH.

? Use of drug as poison was far more common in Kathmandu.

Over the counter sale of the 'prescription only drugs' should be discouraged. An efficient recording system should be developed to record the transaction of the 'prescription only drugs' like name of the prescriber, amount sold etc. The drugs that are liable to be used as poison should be moved to the 'prescription only drugs' category. Department of drug Administration should regularly monitor the retailers and dispensaries.
Below 25 years was the most affected age group at TUTH and BDH respectively. Public should be sensitized about the childhood poisoning and they should be educated about the preventive measures such as keeping the toxic substances, kerosene and drugs out of the reach of the children.

Pesticides and rodenticides were the mostly used poison.

Packaging of drugs and poisons should be designed with safety in mind and with printed warnings.

\section{ACKNOWLEDGEMENT}

Our sincere thank goes to NHRC for providing us the financial support for our research. We are grateful to Prof. Dr Gopal Prasad Acharya, Dr Anand Ballav Joshi, Mr Shyam Lohani and Mr. Sitaram Khakurel who always inspired us and showed us the appropriate way throughout the research to make this research a success.

And thanks to all who helped us get access to the file reports of poisonings in TUTH and BDH.

\section{REFERENCES}

1. Lawson. A.A.H. Acute Poisoning, Epidemiology, Davidson's Principles and Practice of Medicine (17 th Ed.) Churchill Livingstone, 1996: 1126.

2. HMG of Nepal Central bureau of statistics, National Planning Cormission. The Statistical Year book of Nepal. 2001.

3. Pokhrel N., Gurung C.K. A study of poison cases recorded in Bir Hospital over four years. J. Inst. Med. 1987; 9:29-34.

4. Kafle K.K. et.al, Poisoning cases at TU Teaching Hospital, J. Inst. Med, 1989; 11, 297-301.

5. Rauniar G.P et.al , Retrospective Analysis of profile of acute poisoning cases in a tertiary care hospital in eastern Nepal: A four year data base from 1994 to 1997, J. Nep. Med. Assoc. 1999; 38: 23-28.

6. Pathak U.N et.al, Retrospective study of poisoning cases admitted in Nepal Medical college Teaching Hospital, Nepal Medical college Journal 2001; 3 (2) : 101-105.

7. Prasad P.N, Karki prakash, Poisoning cases at TUTH Emergency: a one year review, J. Inst. Med. 1997; 19: 18-24.

8. Zine KU , Mohanty , AZ; Pattern of acute poisoning of Indira Gandhi Medical College and Hospital, Joumal of Indian Academy of Forensic Medicine; 1998 apr-sep; 20 (2) : 37-39.

9. Subedi BK.A Retrospective study of poisoning cases at Bir Hospital Nepal. J. Inst. Med1990; 13 (4) : 296-302.

10. Shrestha B.M, Childhood poisoning analysis and review, J. Inst. Med 1991; 13: 243-256. 